\title{
Drawing Planar Graphs with Reduced Height
}

\author{
Stephane Durocher* and Debajyoti Mondal ${ }^{\star \star}$ \\ Department of Computer Science, University of Manitoba, Canada \\ \{durocher, jyoti\}@cs.umanitoba.ca
}

\begin{abstract}
A straight-line (respectively, polyline) drawing $\Gamma$ of a planar graph $G$ on a set $L_{k}$ of $k$ parallel lines is a planar drawing that maps each vertex of $G$ to a distinct point on $L_{k}$ and each edge of $G$ to a straight line segment (respectively, a polygonal chain with the bends on $L_{k}$ ) between its endpoints. The height of $\Gamma$ is $k$, i.e., the number of lines used in the drawing. In this paper we compute new upper bounds on the height of polyline drawings of planar graphs using planar separators. Specifically, we show that every $n$-vertex planar graph with maximum degree $\Delta$, having a simple cycle separator of size $\lambda$, admits a polyline drawing with height $4 n / 9+O(\lambda \Delta)$, where the previously best known bound was $2 n / 3$. Since $\lambda \in O(\sqrt{n})$, this implies the existence of a drawing of height at most $4 n / 9+o(n)$ for any planar triangulation with $\Delta \in o(\sqrt{n})$. For $n$-vertex planar 3 -trees, we compute straight-line drawings with height $4 n / 9+O(1)$, which improves the previously best known upper bound of $n / 2$. All these results can be viewed as an initial step towards compact drawings of planar triangulations via choosing a suitable embedding of the input graph.
\end{abstract}

\section{Introduction}

A polyline drawing of a planar graph $G$ is a planar drawing of $G$ such that each vertex of $G$ is mapped to a distinct point in the Euclidean plane, and each edge is mapped to a polygonal chain between its endpoints. Let $L_{k}=\left\{l_{1}, l_{2}, \ldots, l_{k}\right\}$ be a set of $k$ horizontal lines such that for each $i \leq k$, line $l_{i}$ passes through the point $(0, i)$. A polyline drawing of $G$ is called a polyline drawing on $L_{k}$ if the vertices and bends of the drawing lie on the lines of $L_{k}$. The height of such a drawing is $k$, i.e., the number of parallel horizontal lines used by the drawing. Such a drawing is also referred to as a $k$-layer drawing in the literature 1318 . Let $\Gamma$ be a polyline drawing of $G$. We call $\Gamma$ a $t$-bend polyline drawing if each of its edges has at most $t$ bends. Thus a 0-bend polyline drawing is also known as a straight-line drawing. Drawing planar graphs on a small integer grid is an active research area in graph drawing [716], which is motivated by the need of compact layout of VLSI circuits and visualization of software architecture. Since simultaneously optimizing the

\footnotetext{
* Work of the author is supported in part by the Natural Sciences and Engineering Research Council of Canada (NSERC).

** Work of the author is supported in part by a University of Manitoba Graduate Fellowship.
} 
width and height of the drawing is very challenging, researchers have also focused their attention on optimizing one dimension of the drawing 6111317, while the other dimension is unbounded. In this paper we develop new techniques that can produce drawings with small height. We distinguish between the terms 'plane' and 'planar'. A plane graph is a planar graph with a fixed combinatorial embedding and a specified outer face. While drawing a planar graph, we allow the output to represent any planar embedding of the graph. On the other hand, while drawing a plane graph, the output is further constrained to respect the input embedding.

State-of-the-art algorithms that compute straight-line drawings of $n$-vertex plane graphs on an $(O(n) \times 2 n / 3)$-size grid imply an upper bound of $2 n / 3$ on the height of straight-line drawings [56]. This bound is tight for plane graphs, i.e., there exist $n$-vertex plane graphs such as plane nested triangles graphs and some plane 3 -trees that require a height of $2 n / 3$ in any of their straightline drawings. Recall that an $n$-vertex nested triangles graph is a plane graph formed by a sequence of $n / 3$ vertex disjoint cycles, $\mathrm{C}_{1}, \mathrm{C}_{2}, \ldots, \mathrm{C}_{n / 3}$, where for each $i \in\{2, \ldots, n / 3\}$, cycle $\mathrm{C}_{i}$ contains the cycles $\mathrm{C}_{1}, \ldots, \mathrm{C}_{i-1}$ in its interior, and a set of edges that connect each vertex of $\mathrm{C}_{i}$ to a distinct vertex in $\mathrm{C}_{i-1}$. Besides, a plane 3 -tree is a triangulated plane graph that can be constructed by starting with a triangle, and then repeatedly adding a vertex to some inner face of the current graph and triangulating that face.

The $2 n / 3$ upper bound on the height is also the currently best known bound for polyline drawings, even for planar graphs, i.e., when we are allowed to choose a suitable embedding for the output drawing. Frati and Patrignani [10] showed that in the variable embedding setting, an $n$-vertex nested triangles graph can be drawn with height at most $n / 3+O(1)$, which is significantly smaller than the lower bound of $2 n / 3$ in the fixed embedding setting. Similarly, Hossain et al. 11] showed that an universal set of $n / 2$ horizontal lines can support all $n$ vertex planar 3-trees, i.e., every planar 3-tree admits a drawing with height at most $n / 2$. They also showed that $4 n / 9$ lines suffice for some subclasses of planar 3 -trees, and asked whether $4 n / 9$ is indeed an upper bound for planar 3-trees.

In the context of optimization, Dujmović et al. 9] gave fixed-parametertractable (FPT) algorithms, parameterized by pathwidth, to decide whether a planar graph admits a straight-line drawing on $k$ horizontal lines. Drawings with minimum number of parallel lines have been achieved for trees [13. Recently, Biedl [2] gave an algorithm to approximate the height of straight-line drawings of 2-connected outerplanar graphs within a factor of 4 .

Contributions. In this paper we show that every $n$-vertex planar graph with maximum degree $\Delta$, having a simple cycle separator of size $\lambda$, admits a drawing with height $4 n / 9+O(\lambda \Delta)$, which is better than the previously best known bound of $2 n / 3$ for any $\lambda \Delta \in o(n)$. This result is an outcome of a new application of the planar separator theorem [8]. Although the technique is simple, it has the potential to be a powerful tool while computing compact drawings for planar triangulations in the variable embedding setting. If the input graphs are restricted to planar 3 -trees, then we can improve the upper bound to $4 n / 9+O(1)$, which 
settles the question of Hossain et al. 11. Furthermore, the drawing we construct in this case is a straight-line drawing.

\section{Preliminary Definitions and Results}

Let $G$ be an $n$-vertex triangulated plane graph. A simple cycle $C$ in $G$ is called a cycle separator if the interior and the exterior of $C$ each contains at most $2 n / 3$ vertices. Let $v_{1}, v_{n}$ and $v_{2}$ be the outer vertices of $G$ in clockwise order on the outer face. Let $\sigma=\left(v_{1}, v_{2}, \ldots, v_{n}\right)$ be an ordering of all vertices of $G$. By $G_{k}$, $2 \leq k \leq n$, we denote the subgraph of $G$ induced by $v_{1} \cup v_{2} \cup \ldots \cup v_{k}$. For each $G_{k}$, the notation $P_{k}$ denotes the path (while walking clockwise) on the outer face of $G_{k}$ that starts at $v_{1}$ and ends at $v_{2}$. We call $\sigma$ a canonical ordering of $G$ with respect to the outer edge $\left(v_{1}, v_{2}\right)$ if for each $k, 3 \leq k \leq n$, the following conditions are satisfied [7]:

(a) $G_{k}$ is 2-connected and internally triangulated.

(b) If $k \leq n$, then $v_{k}$ is an outer vertex of $G_{k}$ and the neighbors of $v_{k}$ in $G_{k-1}$ are consecutive on $P_{k-1}$.

Let $P_{k}$, for some $k \in\{3,4, \ldots, n\}$, be the path $w_{1}\left(=v_{1}\right), \ldots, w_{l}, v_{k}\left(=w_{l+1}\right)$, $w_{r}, \ldots, w_{t}\left(=v_{2}\right)$. The edges $\left(w_{l}, v_{k}\right)$ and $\left(v_{k}, w_{r}\right)$ are the l-edge and $r$-edge of $v_{k}$, respectively. The other edges incident to $v_{k}$ in $G_{k}$ are called the $m$-edges. For example, in Figure 1(c), the edges $\left(v_{6}, v_{1}\right),\left(v_{6}, v_{4}\right)$, and $\left(v_{5}, v_{6}\right)$ are the $l-, r$ and $m$-edges of $v_{6}$, respectively. Let $E_{m}$ be the set of all $m$-edges in $G$. Then the graph $T_{v_{n}}$ induced by the edges in $E_{m}$ is a tree with root $v_{n}$. Similarly, the graph $T_{v_{1}}$ induced by all $l$-edges except $\left(v_{1}, v_{n}\right)$ is a tree rooted at $v_{1}$ (Figure 1(b)), and the graph $T_{v_{2}}$ induced by all $r$-edges except $\left(v_{2}, v_{n}\right)$ is a tree rooted at $v_{2}$. These three trees form the Schnyder realizer [16] of $G$.

Lemma 1 (Bonichon et al. [4]). The total number of leaves in all the trees in any Schnyder realizer of an $n$-vertex triangulation is at most $2 n-5$.

Let $G$ be a planar graph and let $\Gamma$ be a straight-line drawing on $k$ parallel lines. By $l(v)$, where $v$ is a vertex of $G$, we denote the horizontal line in $\Gamma$ that passes through $v$. We now have the following lemma that bounds the height of a straight-line drawing in terms of the number of leaves in a Schnyder tree. The

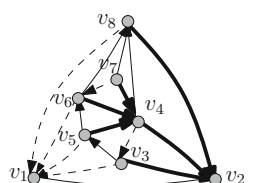

(a)

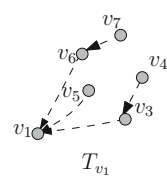

(b)

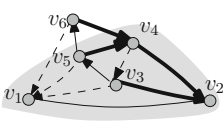

(c)

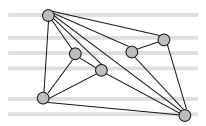

(d)

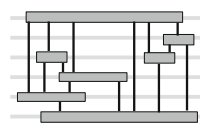

(e)

Fig. 1. (a) A plane triangulation $G$ with a canonical ordering. The associated realizer, where the $l$-, $r$ - and $m$ - edges are shown in dashed, bold-solid, and thin-solid lines, respectively. (b) $T_{v_{1}}$. (c) Neighbors of $v_{6}$ in $G_{6}$. (d)-(e) Illustrating Lemma 3 . 
lemma can be derived from the known straight-line [5] and polyline drawing algorithms 4]. We omit the proof due to space constraints.

Lemma 2. Let $G$ be an n-vertex plane triangulation and let $v_{1}, v_{n}, v_{2}$ be the outer vertices of $G$ in clockwise order on the outer face. Assume that $T_{v_{n}}$ has at most $p$ leaves. Then for any placement of $v_{n}$ on line $l_{1}$ or $l_{p+2}$, there exists a straight-line drawing $\Gamma$ of $G$ on $L_{p+2}$ such that $v_{2}$ and $v_{1}$ lie on lines $l_{p+2}$ and $l_{1}$, respectively.

Chrobak and Nakano [6] showed that every planar graph admits a straightline drawing with height $2 n / 3$. We now observe some properties of Chrobak and Nakano's algorithm [6]. Let $G$ be a plane triangulation with $n$ vertices and let $x, y$ be two user prescribed outer vertices of $G$ in clockwise order. Let $\Gamma$ be the drawing of $G$ produced by the Algorithm of Chrobak and Nakano [6]. Then $\Gamma$ has the following properties:

$\left(\mathrm{CN}_{1}\right) \Gamma$ is a drawing on $L_{q}$, where $q \leq 2 n / 3$.

$\left(\mathrm{CN}_{2}\right)$ For the vertices $x$ and $y$, we have $l(x)=l_{1}$ and $l(y)=l_{q}$ in $\Gamma$. The remaining outer vertex $z$ lies on either $l_{1}$ or $l_{q}$.

Note that the user cannot choose the placement of $z$, i.e., the algorithm may produce a drawing where $l(x)=l_{1}, l(y)=l_{q}$ and $l(z)=l_{1}$, however, this does not imply that there exists another drawing where $l(x)=l_{1}, l(y)=l_{q}$ and $l(z)=l_{q}$. We end this section with the following lemma.

Lemma 3. Let $G$ be a plane graph and let $\Gamma$ be a straight-line drawing of $G$ on $k$ horizontal lines, but the lines are not necessarily equally spaced. Then there exists a drawing $\Gamma^{\prime}$ of $G$ on a set of $k$ horizontal lines that are equally spaced. Furthermore, for every $i \in\{1,2, \ldots, k\}$, the left to right order of the vertices on the ith line in $\Gamma$ coincides with that of $\Gamma^{\prime}$.

Proof (Outline). One can construct $\Gamma^{\prime}$ by first transforming $\Gamma$ into a 'flatvisibility representation' on equally spaced horizontal lines, as shown in Figures 1(d)-(e), and then transforming this representation again into a straightline drawing 113 .

In the following sections we describe our drawing algorithms. Note that for simplicity we often omit the floor and ceiling functions while defining different parameters of the algorithms. One can describe a more careful computation using proper floor and ceiling functions, but that does not affect the asymptotic results discussed in this paper.

\section{Drawing Triangulations with Small Height}

Let $G=(V, E)$ be an $n$-vertex planar triangulation and let $\Gamma$ be a planar drawing of $G$ on the Euclidean plane. Let $C=\left(V_{c}, E_{c}\right)$ be a simple cycle separator of $G$ of size $\lambda$. Let $G_{i}=\left(V_{i}, E_{i}\right)$ be the graph induced by the vertices that lie inside $C$ and on the boundary of $C$. Similarly, let $G_{o}=\left(V_{o}, E_{o}\right)$ be the graph induced by the vertices that lie outside $C$ and on the boundary of $C$. Specifically, $V=V_{i} \cup V_{o}, E=E_{i} \cup E_{o}, V_{i} \cap V_{o}=V_{c}$, and $E_{i} \cap E_{o}=E_{c}$. We now compute a polyline drawing of $G$. 


\subsection{Drawing Technique}

If any edge $(a, b) \in E_{c}$ lies on the outer face of $\Gamma$, then we will draw $G$ respecting the combinatorial embedding determined by $\Gamma$. Otherwise, there exists an edge $(a, b) \in E_{c}$ such that the face $a, b, c$ with $c \in V_{o}$ does not lie interior to $C$. We redefine $\Gamma$ as the embedding of $G$ obtained by choosing $a, b, c$ as the outer face, as illustrated in Figures 2(a)-(b).

Drawing $\boldsymbol{G}_{\boldsymbol{i}}$. Assume that $x=4 n / 9+2 \lambda / 3+3$. Construct a plane graph $G_{i}^{\prime}$ by taking a copy of $G_{i}$ from $\Gamma$, and then adding a vertex $z$ to the outer face of $G_{i}$ along with the edges $(z, w)$, for all $w \in V_{c}$. Figure $2(\mathrm{c})$ illustrates $G_{i}^{\prime}$. Since $G_{i}$ has at most $(2 n+3 \lambda) / 3$ vertices, we now use the algorithm of Chrobak and Nakano [6] to compute a drawing $\Gamma_{i}$ of $G_{i}^{\prime}$ on $L_{x}$, where $a, b$ lie on $l_{1}, l_{x}$ and $z$ lies on either $l_{1}$ or $l_{x}$. Assume without loss of generality that $z$ is in the right half-plane of the line through $a, b$.

Drawing $\boldsymbol{G}_{\boldsymbol{o}}$. Take a copy of $G_{o}$ from $\Gamma$. Let $u$ be any vertex in $G_{o}$. Then by $d_{o}(u)$ we denote the degree of vertex $u$ in $G_{o}$. Let the cycle $C$ be $a\left(=w_{1}\right), w_{2}, \ldots$, $b\left(=w_{\lambda}\right)$. For each vertex $w_{i} \in V_{c}$, where $1 \leq i \leq \lambda$ and $w_{\lambda+1}=w_{1}$, if $d_{o}\left(w_{i}\right)>3$, then replace $\left(w_{i}, w_{i+1}\right)$ with a path $w_{i}, w_{i}^{1}, w_{i}^{2}, \ldots, w_{i}^{d_{o}\left(w_{i}\right)-3}, w_{i+1}$ of $d_{o}\left(w_{i}\right)-3$ division vertices. Let $u_{1}, u_{2}, \ldots, u_{d_{o}\left(w_{i}\right)-2}$ be the neighbors of $w_{i}$ in clockwise order outside of $C$. Then delete the edges from $w_{i}$ to these neighbors, and add the edges $\left(w_{i}, u_{1}\right),\left(w_{i}^{1}, u_{2}\right), \ldots,\left(w_{i}^{d_{o}\left(w_{i}\right)-3}, u_{d_{o}\left(w_{i}\right)-2}\right)$. Replace the edge $\left(w_{1}, w_{\lambda}^{d_{o}\left(w_{\lambda}\right)-3}\right)$ by a path $w_{1}, w^{\prime}, w^{\prime \prime}, w_{\lambda}^{d_{o}\left(w_{\lambda}\right)-3}$, and redefine $a$ and $b$ such that $a=w^{\prime \prime}$ and $b=w^{\prime}$. Let the resulting graph be $H$ and let the newly constructed cycle be $C^{\prime}$. Figure 2(d) illustrates $H$.

If $z$ lies on $l_{1}$ in $\Gamma_{i}$, then we add the edges $(a, w)$ to $H$, for each vertex $w$ on $C^{\prime}$. Otherwise, we add the edges $(b, w)$ to $H$. Finally, we add a vertex $z^{\prime}$ on the outer face and triangulate $H$ such that $(a, b)$ remains an outer edge. Let the resulting graph be $G_{o}^{\prime}$. Figure 2(e) illustrates $G_{o}^{\prime}$. Observe that the number of vertices in $G_{o}^{\prime}$ is at most $2 n / 3+\lambda \Delta+3$. Hence we can use the algorithm of Chrobak and Nakano [6] to compute a drawing $\Gamma_{o}$ of $G_{o}^{\prime}$ on $L_{y}$, where $y=(4 n+6 \lambda \Delta+18) / 9$, such that $a, b$ lie on $l_{1}, l_{y}$, respectively, and the segment $a b$ is vertical. Assume without loss of generality that all the vertices of $G_{o}^{\prime}$ are in the right half-plane of the line through $a, b$.

Merging $\boldsymbol{G}_{\boldsymbol{i}}$ and $\boldsymbol{G}_{\boldsymbol{o}}$. Without loss of generality assume that $l(z)=l_{x}$ in $\Gamma_{i}$, and recall that in this case $b$ is adjacent to all the vertices on $C^{\prime}$ in $\Gamma_{o}$. Let $\ell_{o}$ be a vertical line to the right of segment $a b$ in $\Gamma_{o}$ such that all the other vertices of $\Gamma_{o}$ are in the right half-plane of $\ell_{o}$. Furthermore, $\ell_{o}$ must be close enough such that all the intersection points with the edges incident to $b$ lie in between the horizontal line through $b$ and the next horizontal line. For each intersection point, we insert a division vertex at that point and create a horizontal line through that vertex. We then delete vertex $b$ from $\Gamma_{o}$, but not the division vertices. Figures 2(i)-(j) illustrate this scenario. By Lemma 3, we can modify $\Gamma_{o}$ such that the horizontal lines are equally spaced. Since $C^{\prime}$ contains at most $\lambda \Delta$ vertices, $\Gamma_{o}$ is a drawing on at most $y+\lambda \Delta$ horizontal lines. Similarly, we modify $\Gamma_{i}$, as follows. 


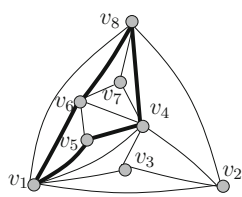

(a)

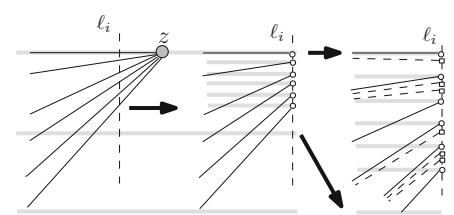

(f)

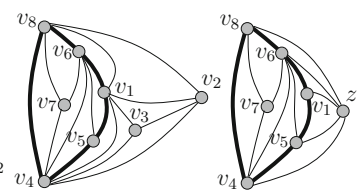

(b)

(g) (c)

(h)

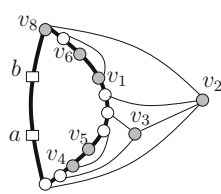

(d)

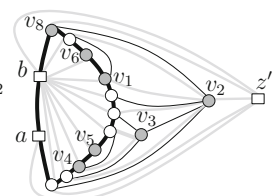

(e)

Fig. 2. (a) A plane triangulation $G$, where $C$ is shown in bold. (b) $\Gamma$, where $v_{4}=$ $a, v_{8}=b$ and $v_{2}=c$. (c) $G_{i}^{\prime}$. (d) $H$, where the division vertices are shown in white. (e) $G_{o}^{\prime}$, where the edges added to $H$ are shown in gray. (f)-(j) Drawing $G$.

Let $\ell_{i}$ be a vertical line to the left of $z$ in $\Gamma_{i}$ such that all the other vertices of $\Gamma_{i}$ are in the left half-plane of $\ell_{i}$. Furthermore, $\ell_{i}$ must be close enough such that all the intersection points with the edges incident to $z$ lie in between two consecutive parallel lines. For each intersection point, we insert a division vertex at that point and create a horizontal line through that vertex. Let $v_{1}, v_{2}, \ldots, v_{\lambda}$ be the division vertices on $\ell_{i}$ in the order of decreasing $y$-coordinate, where for each $i \in\{1,2, \ldots, \lambda\}, v_{i}$ is incident to the vertex $w_{i}$ on $C$. Delete vertex $z$, but not the division vertices. For each vertex $w_{i}$, if $d_{o}\left(w_{i}\right)>3$, then we place a set of division vertices $v_{i}^{1}, v_{i}^{2}, \ldots, v_{i}^{d_{o}\left(w_{i}\right)-3}$ below $v_{i}$ and above the horizontal line closest to $v_{i}$. Besides, these new division vertices must be sufficiently close to $v_{i}$ such that drawing of the edges $\left(w_{i}, v_{i}^{j}\right)$, where $1 \leq j \leq d_{o}\left(w_{i}\right)-3$, do not create any edge crossing. Figures 2 (f) - (h) illustrate this scenario. Finally, by Lemma 3 . we can modify $\Gamma_{i}$ such that the horizontal lines are equally spaced. Note that $\Gamma_{i}$ is a drawing on at most $x+\lambda \Delta$ horizontal lines.

Since the division vertices in $\Gamma_{i}$ and $\Gamma_{o}$ take a set of consecutive horizontal lines from their respective topmost lines, it is straightforward to merge these two drawings on a set of $\lambda \Delta+\max \{x, y\}=4 n / 9+O(\lambda \Delta)$ horizontal lines. We delete the edges on $C^{\prime}$, and consider all vertices of $C^{\prime}$ as division vertices. Since the division vertices correspond to the bends, each edge may contain at most six bends (two bends to enter $\Gamma_{o}$ from $\Gamma_{i}$, two bends on $C^{\prime}$, and two bends to return to $\Gamma_{i}$ from $\Gamma_{o}$ ). Since there are at most $\lambda \Delta$ edges that may have bends, the number of bends is at most $6 \lambda \Delta$ in total. However, via 'flat-visibility representation' (similar to the proof of Lemma 3) one can reduce the number of bends to enter and exit $\Gamma_{o}$ by one, we omit the details due to space constraints. Hence the number of bends reduces to $4 \lambda \Delta$. The following theorem summarizes the result of this section. 
Theorem 1. Let $G$ be an n-vertex planar graph with maximum degree $\Delta$. If $G$ contains a simple cycle separator of size $\lambda$, then $G$ admits a 4-bend polyline drawing with height $4 n / 9+O(\lambda \Delta)$ and at most $4 \lambda \Delta$ bends in total.

Since every planar triangulation has a simple cycle separator of size $O(\sqrt{n})[8$, we obtain the following corollary.

Corollary 1. Every $n$-vertex planar triangulation with maximum degree $o(\sqrt{n})$ admits a polyline drawing with height at most $4 n / 9+o(n)$.

Pach and Tóth [15] showed that polyline drawings can be transformed into straight-line drawings while preserving the height if the polyline drawing is monotone, i.e., if every edge in the polyline drawing is drawn as a $y$-monotone curve. Unfortunately, our algorithm does not necessarily produce monotone drawings.

\section{Drawing Planar 3-Trees with Small Height}

In this section we examine straight-line drawings of planar 3-trees.

\subsection{Technical Background}

Let $G$ be an $n$-vertex planar 3 -tree and let $\Gamma$ be a straight-line drawing of $G$. Then $\Gamma$ can be constructed by starting with a triangle, which corresponds to the outer face of $\Gamma$, and then iteratively inserting the other vertices into the inner faces and triangulating the resulting graph. Let $a, b, c$ be the outer vertices of $\Gamma$ in clockwise order. If $n>3$, then $\Gamma$ has a unique vertex $p$ that is incident to all the outer vertices. This vertex $p$ is called the representative vertex of $G$.

For any cycle $i, j, k$ in $G$, let $G_{i j k}$ be the subgraph induced by the vertices $i, j, k$ and the vertices lying inside the cycle. Let $G_{i j k}^{*}$ be the number of vertices in $G_{i j k}$. The following two lemmas describe some known results.

Lemma 4 (Mondal et al. 14]). Let $G$ be a plane 3-tree and let $i, j, k$ be a cycle of three vertices in $G$. Then $G_{i j k}$ is a plane 3 -tree.

Lemma 5 (Hossain et al. [11]). Let $G$ be an n-vertex plane 3-tree with the outer vertices $a, b, c$ in clockwise order. Let $D$ be a drawing of the outer cycle $a, b, c$ on $L_{n}$, where the vertices lie on $l_{1}, l_{k}$ and $l_{i}$ with $k \leq n$ and $i \in\left\{l_{1}, l_{2}, l_{n}, l_{n-1}\right\}$. Then $G$ admits a straight-line drawing $\Gamma$ on $L_{k}$, where the outer cycle of $\Gamma$ coincides with $D$.

Let $G$ be a plane 3 -tree and let $a, b, c$ be the outer vertices of $G$. Assume that $G$ has a drawing $\Gamma$ on $L_{k}$, where $a, b$ lie on lines $l_{1}, l_{k}$, respectively, and $c$ lies on line $l_{i}$, where $1 \leq i \leq k$. Then the following properties hold for $\Gamma$ [1].

Reshape. Let $p, q$ and $r$ be three different points on lines $l_{1}, l_{k}$ and $l_{i}$, respectively. Then $G$ has a drawing $\Gamma^{\prime}$ on $L_{k}$ such that the outer face of $\Gamma^{\prime}$ coincides with triangle pqr (e.g., Figures $\underline{3}$ (a)-(b)). 
Stretch. For any integer $t \geq k, G$ admits a drawing $\Gamma^{\prime}$ on $L_{t}$ such that $a, b, c$ lie on $l_{1}, l_{t}, l_{i}$, respectively (e.g., Figure $3(\mathrm{c})$ ).

For any triangulation $H$ with the outer vertices $a, b, c$, let $T_{a, H}, T_{b, H}, T_{c, H}$ be the Schnyder trees rooted at $a, b, c$, respectively. By leaf $(T)$ we denote the number of leaves in $T$. The following lemma establishes a sufficient condition for a plane 3 -tree $G$ to have a straight-line drawing with height at most $4(n+3) / 9+4$.

Lemma 6. Let $G$ be an $n$-vertex plane 3 -tree with outer vertices $a, b, c$ in clockwise order. Let $w_{1}, \ldots, w_{k}(=p), w_{k+1}(=q), \ldots, w_{t}(=c)$ be the maximal path $P$ such that each vertex on $P$ is adjacent to both $a$ and $b$. Assume that $n^{\prime}=n+3$, and $x=4 n^{\prime} / 9$. If $G_{a p q}^{*} \leq\left(n^{\prime}+2\right) / 3, G_{b p q}^{*} \leq G_{a b p}^{*} \leq n^{\prime} / 2$ and $\max _{\forall i>k+1}\left\{G_{a w_{i} w_{i-1}}^{*}, G_{b w_{i} w_{i-1}}^{*}\right\} \leq 4 n^{\prime} / 9$, then $G$ admits a drawing with height at most $4 n^{\prime} / 9+4$.

Proof. Let $H$ be the subgraph of $G$ induced by the vertices $\{a, b\} \cup\left\{w_{k}, \ldots, w_{t}\right\}$. The idea of the proof is first to construct a drawing of $H$ on $L_{x+4}$, and then to extend it to the required drawing using Lemmas 2 25. We distinguish two cases depending on whether leaf $\left(T_{p, G_{a b p}}\right) \leq x$ or not.

Case $1\left(\operatorname{leaf}\left(\boldsymbol{T}_{\boldsymbol{p}, \boldsymbol{G}_{a b p}}\right) \leq \boldsymbol{x}\right)$. Since $G_{b q p}^{*} \leq n^{\prime} / 2$, by Lemma 1, one of the trees in the Schnyder realizer of $G_{b q p}$ has at most $n^{\prime} / 3 \leq x$ leaves. We now draw $G_{a b q}$ considering the following scenarios.

Case $1 \mathrm{~A}\left(\operatorname{leaf}\left(\boldsymbol{T}_{\boldsymbol{p}, \boldsymbol{G}_{b q p}}\right) \leq \boldsymbol{x}\right)$. By Lemma2 2 and the Stretch condition, $G_{a b p}$ admits a drawing $\Gamma_{a b p}$ on $L_{x+2}$ such that the vertices $a, b, p$ lie on $l_{1}, l_{x+2}, l_{x+2}$, respectively. Similarly, $G_{b q p}$ admits a drawing $\Gamma_{b p q}$ on $L_{x+2}$ such that the vertices $q, b, p$ lie on $l_{1}, l_{x+2}, l_{x+2}$, respectively, as shown in Figure 3(d). By the Stretch property, $\Gamma_{a b p}$ can be extended to a drawing $\Gamma_{a b p}^{\prime}$ on $L_{x+3}$, where $a, b, p$ lie on $l_{1}, l_{x+3}, l_{x+2}$, respectively. Similarly, $\Gamma_{b q p}$ can be extended to a drawing $\Gamma_{b q p}^{\prime}$ on $L_{x+3}$, where $q, b, p$ lie on $l_{1}, l_{x+3}, l_{x+2}$, respectively. Since $G_{a p q}^{*} \leq\left(n^{\prime}+2\right) / 3$, by Lemma 5 and the Stretch condition, $G_{a p q}$ admits a drawing $\Gamma_{a p q}$ on $L_{\left(n^{\prime}+2\right) / 3}$. Finally, by the Stretch property $\Gamma_{a p q}$ can be extended to a drawing $\Gamma_{a p q}^{\prime}$ on $L_{x+2}$ such that $a, p, q$ lie on $l_{1}, l_{x+2}, l_{1}$, respectively, and by the Reshape property we can merge these drawings to obtain a drawing of $G_{a b q}$ on $L_{x+3}$. Figure 3(e) depicts an illustration.

Case 1B ( $\left.\operatorname{leaf}\left(\boldsymbol{T}_{\boldsymbol{b}, \boldsymbol{G}_{b q p}}\right) \leq \boldsymbol{x}\right)$. By Lemma 2 and the Stretch condition, $G_{a b p}$ admits a drawing $\Gamma_{a b p}$ on $L_{x+2}$ such that the vertices $a, b, p$ lie on $l_{1}, l_{x+2}, l_{1}$,

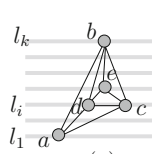

(a)

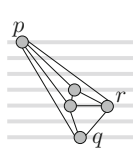

(b)

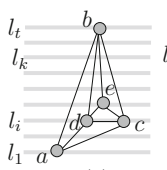

(c)

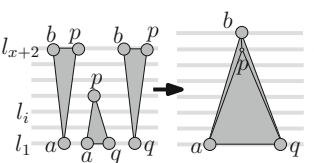

(d) (e)

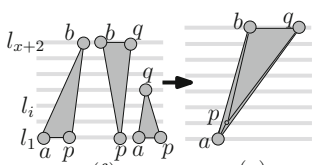

(g)

Fig. 3. (a)-(b) Illustrating Reshape. (c) Illustrating Stretch. (d)-(e) Illustration for Case 1A. (d)-(e) Illustration for Case 1B. 
respectively. Similarly, $G_{b q p}$ admits a drawing $\Gamma_{b p q}$ on $L_{x+2}$ such that the vertices $p, b, q$ lie on $l_{1}, l_{x+2}, l_{x+2}$, respectively. By Lemma 5. $G_{a p q}$ admits a drawing $\Gamma_{a p q}$ on $L_{\left(n^{\prime}+2\right) / 3}$ such that $a, p, q$ lie on $l_{1}, l_{1}, l_{x+2}$, respectively. Finally, by Stretch and Reshape we can merge these drawings to obtain a drawing of $G_{a b q}$ on $L_{x+3}$. Figures $3(\mathrm{f})-(\mathrm{g})$ show an illustration.

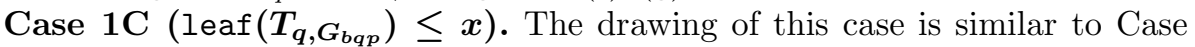
1B. The only difference is that we use $T_{q, G_{b q p}}$ while drawing $G_{b q p}$.

Observe that each of the Cases $1 \mathrm{~A}-1 \mathrm{C}$ produces a drawing of $G_{a b q}$ such that $a, b$ lie on $l_{1}, l_{x+3}$, respectively, and $q$ lies on either $l_{1}$ or $l_{x+3}$. We stretch it to a drawing on $L_{x+4}$ such that $a, b$ lies on $l_{1}, l_{x+4}$, respectively, and $q$ lies on either $l_{2}$ or $l_{x+3}$. If $q$ lies on $l_{2}$, then we draw the path $w_{k+1}, \ldots, w_{t}(=c)$ in a zigzag fashion, placing the vertices on $l_{2}$ and $l_{x+3}$ alternatively such that each vertex is visible to both $a$ and $b$. Similarly, if $q$ lies on $l_{x+3}$, then we place the vertices $w_{k+1}, \ldots, w_{t}(=c)$ on $l_{x+3}$ and $l_{2}$ alternatively, as shown in Figure 4(a). For each $i>k+1$, Lemma 4 ensures that the graphs $G_{a w_{i} w_{i-1}}$ and $G_{b w_{i} w_{i-1}}$ are plane 3 -trees. Since $\max _{\forall i>k+1}\left\{G_{a w_{i} w_{i-1}}^{*}, G_{b w_{i} w_{i-1}}^{*}\right\} \leq x$, we can draw $G_{a w_{i} w_{i-1}}$ and $G_{b w_{i} w_{i-1}}$ using Lemma [5 inside their corresponding triangles.

Case $2\left(\operatorname{leaf}\left(\boldsymbol{T}_{\boldsymbol{p}, \boldsymbol{G}_{a b p}}\right)>\boldsymbol{x}\right)$. Since $G_{a b p}^{*} \leq n^{\prime} / 2$, by Lemma 1, leaf $\left(T_{a, G_{a b p}}\right)+$ $\operatorname{leaf}\left(T_{b, G_{a b p}}\right) \leq n^{\prime}-\operatorname{leaf}\left(T_{p, G_{a b p}}\right) \leq 5 n^{\prime} / 9$. Hence we draw $G_{a b q}$ considering the following scenarios.

Case 2A (leaf $\left(\boldsymbol{T}_{a, G_{a b p}}\right) \leq \boldsymbol{x}$ and $\left.\operatorname{leaf}\left(\boldsymbol{T}_{\boldsymbol{b}, \boldsymbol{G}_{a b p}}\right) \leq \boldsymbol{x}\right)$. Since $G_{b q p}^{*} \leq n^{\prime} / 2$, by Lemma 1, one of the trees in the Schnyder realizer of $G_{b q p}$ has at most $n^{\prime} / 3 \leq x$ leaves. If $\operatorname{leaf}\left(T_{p, G_{b p q}}\right) \leq x$, then we draw $G_{a b q}$ on $L_{x+3}$, where $a, b, p, q$ lie on $l_{1}, l_{x+3}, l_{x+2}, l_{1}$, respectively, as in Figure 4(b). Otherwise, either leaf $\left(T_{b, G_{b p q}}\right) \leq x$ or $\operatorname{leaf}\left(T_{q, G_{b p q}}\right) \leq x$. In this case we draw $G_{a b q}$ on $L_{x+3}$, where $a, b, p, q$ lie on $l_{1}, l_{x+3}, l_{2}, l_{x+3}$, respectively, as in Figure 4 (c).

Case 2B $\left(\operatorname{leaf}\left(\boldsymbol{T}_{\boldsymbol{a}, \boldsymbol{G}_{a b p}}\right)>\boldsymbol{x}\right.$ and $\left.\operatorname{leaf}\left(\boldsymbol{T}_{\boldsymbol{b}, \boldsymbol{G}_{\boldsymbol{a b p}}}\right) \leq \boldsymbol{n}^{\prime} / \mathbf{9}\right)$. If $\operatorname{leaf}\left(T_{p, G_{b p q}}\right) \leq$ $n^{\prime} / 3$, then we first draw $G_{b p q}$ using Lemma 2 such that $b, p, q$ lie on $l_{n^{\prime} / 3+2}$, $l_{n^{\prime} / 3+2}, l_{1}$, respectively, and then use the Stretch condition to shift $b$ to $l_{x+3}$. By Lemma 2 and the Stretch condition, there exists a drawing of $G_{a b p}$ on $L_{x+3}$ with $a, b, p$ lying on $l_{1}, l_{x+3}, l_{n^{\prime} / 3+2}$, respectively. Since $G_{a p q}^{*} \leq\left(n^{\prime}+\right.$ 2)/3, we can draw $G_{a p q}$ using Lemma 5 inside triangle apq. Figure 4 (d) illustrates the scenario after applying Stretch and Reshape.

If leaf $\left(T_{p, G_{b p q}}\right)>n^{\prime} / 3$, then by Lemma 1 either leaf $\left(T_{b, G_{b p q}}\right) \leq n^{\prime} / 3$ or $\operatorname{leaf}\left(T_{q, G_{b p q}}\right) \leq n^{\prime} / 3$. Hence we can use Lemma 2 and the Stretch condition

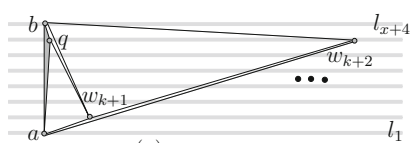

(a)

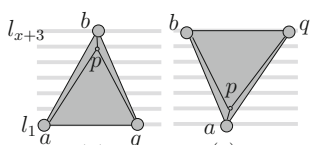

(b)

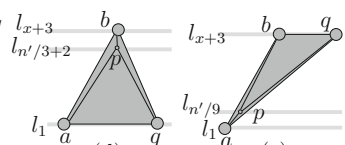

(d) (e)

Fig. 4. (a) Illustrating Case 1. (b)-(c) Illustrating Case 2A. (d)-(e) Case 2B. 
to draw $G_{b p q}$ such that $b, p, q$ lie on $l_{x+3}, l_{n^{\prime} / 9}, l_{x+3}$, respectively. On the other hand, we use Lemma2 2 and the Stretch condition to draw $G_{a b p}$ such that $a, b, p$ lie on $l_{1}, l_{x+3}, l_{n^{\prime} / 9}$, respectively. Since $G_{a p q}^{*} \leq\left(n^{\prime}+2\right) / 3$, we can draw $G_{a p q}$ using Lemma 5 inside triangle $a p q$. Figure $4(\mathrm{e})$ illustrates the scenario.

Case 2C (leaf $\left(T_{a, G_{a b p}}\right) \leq \boldsymbol{n}^{\prime} / \mathbf{9}$ and leaf $\left(\boldsymbol{T}_{\left.\boldsymbol{b}, \boldsymbol{G}_{a b p}\right)}>\boldsymbol{x}\right)$. Since each of the edges among $(a, b)$ and $(b, p)$ spans at least $n^{\prime} / 9+2$ parallel lines in Case $2 \mathrm{~B}$, the drawing in this case is analogous to Case $2 \mathrm{~B}$. The only difference is that we use $T_{a, G_{a b p}}$ while drawing $G_{a b p}$.

Each of the Cases $2 \mathrm{~A}-2 \mathrm{C}$ produces a drawing of $G_{a b q}$ such that $a, b$ lies on $l_{1}, l_{x+3}$, respectively, and $q$ lies on either $l_{1}$ or $l_{x+3}$. Hence we can extend these drawings to draw $G$ as in Case 1.

\subsection{Drawing Algorithm}

Decomposition. Let $G$ be an $n$-vertex plane 3 -tree with the outer vertices $a, b, c$ and the representative vertex $p$. A tree spanning the inner vertices of $G$ is called the representative tree $T$ if it satisfies the following conditions [14:

(a) If $n=3$, then $T$ is empty.

(b) If $n=4$, then $T$ consists of a single vertex.

(c) If $n>4$, then the root $p$ of $T$ is the representative vertex of $G$ and the subtrees rooted at the three clockwise ordered children $p_{1}, p_{2}$ and $p_{3}$ of $p$ in $T$ are the representative trees of $G_{a b p}, G_{b c p}$ and $G_{c a p}$, respectively.

Recall that every $n$-vertex tree $T^{\prime}$ has a vertex $v^{\prime}$ such that the connected components of $T^{\prime} \backslash v^{\prime}$ are all of size at most $n / 2$ [12. Such a vertex $v$ in $T$ corresponds to a decomposition of $G$ into four smaller plane 3 -trees $G_{1}, G_{2}, G_{3}$, and $G_{4}$, as follows.

- The plane 3 -tree $G_{i}$, where $1 \leq i \leq 3$, is determined by the representative tree rooted at the $i$ th child of $v$, and thus contains at most $(n+3) / 2$ vertices.

- The plane 3 -tree $G_{4}$ is obtained by deleting $v$ and the vertices from $G$ that are descendent of $v$ in $T$, and contains at most $(n+3) / 2$ vertices.

Drawing Technique. Without loss generality assume that $G_{3}^{*} \leq G_{2}^{*} \leq G_{1}^{*}$. If $G_{1}$ is incident to the outer face of $G$, then let $(a, b)$ be the corresponding outer edge. Otherwise, $G_{1}$ does not have any edge incident to the outer face of $G$. In this case there exists an inner face $f$ in $G$ that is incident to $G_{1}$, but does not belong to $G_{1}$. We choose $f$ as the outer face of $G$, and now we have an edge $(a, b)$ of $G_{1}$ that is incident to the outer face. Let $P=\left(w_{1}, \ldots, w_{k}(=p), w_{k+1}(=q), \ldots, w_{t}\right)$ be the maximal path in $G$ such that each vertex on $P$ is adjacent to both $a$ and $b$, where $\{a, b, p\},\{a, p, q\},\{b, q, p\}$ are the outer vertices of $G_{1}, G_{2}, G_{3}$, respectively. Assume that $n^{\prime}=n+3$ and $x=4 n^{\prime} / 9$. We draw $G$ on $L_{x+4}$ by distinguishing two cases depending on whether $G_{4}^{*}>x$ or not.

Case $1\left(G_{4}^{*}>\boldsymbol{x}\right)$. Observe that $G_{2}^{*} \leq G_{1}^{*} \leq n^{\prime} / 2$ and since $G_{3}^{*}+G_{2}^{*}+G_{1}^{*} \leq$ $n^{\prime}+5-G_{4}^{*}$, we have $G_{3}^{*} \leq 5 n^{\prime} / 27+5 / 3 \leq n^{\prime} / 3$ for sufficiently large values of $n$. 
If $\max _{\forall i>k+1}\left\{G_{a w_{i} w_{i-1}}^{*}, G_{b w_{i} w_{i-1}}^{*}\right\} \leq x$ holds, then $G$ admits a drawing on $L_{x+4}$ by Lemma 6. We may thus assume that there exists some $j>q$ such that either $G_{a w_{j} w_{j-1}}^{*}>x$ or $G_{b w_{j} w_{j-1}}^{*}>x$. Hence $\max _{\forall i>k+1, i \neq j}\left\{G_{a w_{i} w_{i-1}}^{*}, G_{b w_{i} w_{i-1}}^{*}\right\} \leq$ $n^{\prime} / 9$.

We first show that $G_{a b q}$ can be drawn on $L_{x+3}$ in two ways: One drawing $\Gamma_{1}$ contains the vertices $a, b, q$ on $l_{1}, l_{x+3}, l_{2}$, respectively, and the other drawing $\Gamma_{2}$ contains $a, b, q$ on $l_{1}, l_{x+3}, l_{x+2}$, respectively. We then extend these drawings to obtain the required drawing of $G$. Consider the following scenarios depending on whether $G_{1}^{*} \leq x$ or not.

- If $G_{1}^{*} \leq x$, then $G_{3}^{*} \leq G_{2}^{*} \leq G_{1}^{*} \leq x$. Here we draw the subgraph $G^{\prime}$ induced by the vertices $a, b, p, q$ such that they lie on $l_{1}, l_{x+3}, l_{x+2}, l_{2}$, respectively. Since $G_{3}^{*} \leq G_{2}^{*} \leq G_{1}^{*} \leq x$, by Lemma 5, $G_{1}, G_{2}$ and $G_{3}$ can be drawn inside their corresponding triangles, which corresponds to $\Gamma_{1}$. Similarly, we can find another drawing $\Gamma_{2}$ of $G_{a b q}$, where the vertices $a, b, p, q$ lie on $l_{1}, l_{x+3}, l_{2}, l_{x+2}$, respectively. - If $G_{1}^{*}>x$, then $G_{3}^{*} \leq G_{2}^{*} \leq n^{\prime} / 9$. We use Chrobak and Nakano's algorithm [6] and the Stretch condition to draw $G_{1}$ on $L_{x+3}$ layers such that $a, b$ lie on $l_{1}, l_{x+3}$, respectively, and $p$ lies either on $l_{2}$ or $l_{n^{\prime} / 3+2}$. If $l(p)=l_{2}$ (i.e., $\Gamma_{2}$ ), then we place $q$ on $l_{x+2}$. Otherwise, $l(p)=l_{n^{\prime} / 3+2}$ (i.e., $\Gamma_{1}$ ), and we place $q$ on $l_{2}$. Since $G_{3}^{*} \leq G_{2}^{*} \leq n^{\prime} / 9$, for each of these two placements we can draw $G_{2}$ and $G_{3}$ using Lemma 5 inside their corresponding triangles.

We now show how to extend the drawing of $G_{a b q}$ to compute the drawing of $G$. Consider two scenarios depending on whether $G_{a w_{j} w_{j-1}}^{*}>x$ or $G_{b w_{j} w_{j-1}}^{*}>x$.

- Assume that $G_{a w_{j} w_{j-1}}^{*}>x$. Shift $b$ to $l_{x+4}$, and draw the path $w_{k+1}, \ldots, w_{j-1}$ in a zigzag fashion, placing the vertices on $l_{2}$ and $l_{x+3}$ alternatively, such that $l\left(w_{k+1}\right) \neq l\left(w_{k+2}\right)$, and each vertex is visible to both $a$ and $b$. Choose $\Gamma_{1}$ or $\Gamma_{2}$ such that the edge $\left(a, w_{j-1}\right)$ spans at least $x+3$ lines. We now draw $G_{a w_{j} w_{j-1}}$ using Chrobak and Nakano's algorithm [6]. Since $x<G_{a w_{j} w_{j-1}} \leq n^{\prime} / 2$, we can draw $G_{a w_{j} w_{j-1}}$ on at most $n^{\prime} / 3$ parallel lines. By the Stretch and Reshape conditions, we merge this drawing with the current drawing such that $w_{j}$ lies on either $l_{x+3}$ or $l_{n^{\prime} / 9+2}$. Since $G_{b w_{j} w_{j-1}}^{*} \leq n^{\prime} / 9$, we can draw $G_{b w_{j} w_{j-1}}$ inside its corresponding triangle using Lemma 5. Since $\max _{\forall i>j}\left\{G_{a w_{i} w_{i-1}}^{*}, G_{b w_{i} w_{i-1}}^{*}\right\} \leq$ $n^{\prime} / 9$, it is straightforward to extend the current drawing to a drawing of $G$ on $x+4$ parallel lines by continuing the path $w_{j}, \ldots, w_{t}$ in the zigzag fashion.

- Assume that $G_{b w_{j} w_{j-1}}^{*}>x$. The drawing in this case is similar to the case when $G_{a w_{j} w_{j-1}}^{*}>x$. The only difference is that while drawing the path $w_{k+1}, \ldots, w_{j-1}$, we choose $\Gamma_{1}$ or $\Gamma_{2}$ such that the edge $\left(b, w_{j-1}\right)$ spans at least $x+3$ lines.

Case $2\left(\boldsymbol{G}_{4}^{*} \leq \boldsymbol{x}\right)$. Observe that $G_{2}^{*} \leq G_{1}^{*} \leq n^{\prime} / 2$. Since $G_{3}^{*} \leq G_{2}^{*} \leq G_{1}^{*}$ and $G_{3}^{*}+G_{2}^{*}+G_{1}^{*}=n+5$, we have $G_{3}^{*} \leq\left(n^{\prime}+2\right) / 3$. Hence $G$ admits a drawing on $L_{x+4}$ by Lemma 6 .

The following theorem summarizes the result of this section.

Theorem 2. Every n-vertex planar 3-tree admits a straight-line drawing with height $4(n+3) / 9+4=4 n / 9+O(1)$. 
Acknowledgement. We thank the anonymous reviewers for their detailed feedback to improve the presentation of the paper.

\section{References}

1. Biedl, T.: Height-preserving transformations of planar graph drawings. In: Duncan, C., Symvonis, A. (eds.) GD 2014. LNCS, vol. 8871, pp. 380-391. Springer, Heidelberg (2014)

2. Biedl, T.: A 4-approximation for the height of drawing 2-connected outer-planar graphs. In: Erlebach, T., Persiano, G. (eds.) WAOA 2012. LNCS, vol. 7846, pp. 272-285. Springer, Heidelberg (2013)

3. Biedl, T.C.: Transforming planar graph drawings while maintaining height. CoRR abs/1308.6693 (2013), http://arxiv.org/abs/1308.6693

4. Bonichon, N., Le Saëc, B., Mosbah, M.: Wagner's Theorem on Realizers. In: Widmayer, P., Triguero, F., Morales, R., Hennessy, M., Eidenbenz, S., Conejo, R. (eds.) ICALP 2002. LNCS, vol. 2380, pp. 1043-1053. Springer, Heidelberg (2002)

5. Brandenburg, F.J.: Drawing planar graphs on $\frac{8}{9} n^{2}$ area. Electronic Notes in Discrete Mathematics 31, 37-40 (2008)

6. Chrobak, M., Nakano, S.: Minimum width grid drawings of plane graphs. In: Tamassia, R., Tollis, I.G. (eds.) GD 1994. LNCS, vol. 894, pp. 104-110. Springer, Heidelberg (1995)

7. De Fraysseix, H., Pach, J., Pollack, R.: How to draw a planar graph on a grid. Combinatorica 10(1), 41-51 (1990)

8. Djidjev, H., Venkatesan, S.M.: Reduced constants for simple cycle graph separation. Acta Informatica 34(3), 231-243 (1997)

9. Dujmović, V., et al.: On the parameterized complexity of layered graph drawing. In: Meyer auf der Heide, F. (ed.) ESA 2001. LNCS, vol. 2161, pp. 488-499. Springer, Heidelberg (2001)

10. Frati, F., Patrignani, M.: A note on minimum-area straight-line drawings of planar graphs. In: Hong, S.-H., Nishizeki, T., Quan, W. (eds.) GD 2007. LNCS, vol. 4875, pp. 339-344. Springer, Heidelberg (2008)

11. Hossain, M.I., Mondal, D., Rahman, M.S., Salma, S.A.: Universal line-sets for drawing planar 3-trees. Journal of Graph Algorithms and Applications 17(2), 59-79 (2013)

12. Jordan, C.: Sur les assemblages de lignes. Journal für die reine und angewandte Mathematik 70(2), 185-190 (1969)

13. Mondal, D., Alam, M.J., Rahman, M.S.: Minimum-layer drawings of trees (extended abstract). In: Katoh, N., Kumar, A. (eds.) WALCOM 2011. LNCS, vol. 6552, pp. 221-232. Springer, Heidelberg (2011)

14. Mondal, D., Nishat, R.I., Rahman, M.S., Alam, M.J.: Minimum-area drawings of plane 3-trees. Journal of Graph Algorithms and Applications 15(2), 177-204 (2011)

15. Pach, J., Tóth, G.: Monotone drawings of planar graphs. Journal of Graph Theory 46(1), 39-47 (2004)

16. Schnyder, W.: Embedding planar graphs on the grid. In: Proceedings of ACMSIAM SODA, January 22-24, pp. 138-148. ACM (1990)

17. Suderman, M.: Pathwidth and layered drawing of trees. Journal of Computational Geometry \& Applications 14(3), 203-225 (2004)

18. Suderman, M.: Pathwidth and layered drawings of trees. International Journal of Computational Geometry and Applications 14, 203-225 (2004) 